\title{
Preservar ou substituir a patela durante a artroplastia total de joelho: Estudo comparativo
}

\section{To Retain or Resurface the Patella in Primary Total Knee Arthroplasty: A Comparative Study}

\author{
Larry Rodrigues De Campos Júnior ${ }^{1 \oplus}$ Guilherme Norberto Sbalqueiro ${ }^{10}$ \\ Ruben Amancio Rojas Ayala1® Osmar Valadão Lopes Junior ${ }^{1,2(}$ Paulo Renato Fernandes Saggin ${ }^{1,2(1)}$ \\ Andre Kuhn ${ }^{1,2}$,

\footnotetext{
${ }^{1}$ Serviço de Ortopedia e Traumatologia, Hospital São Vicente de Paulo, Passo Fundo, RS, Brasil

${ }^{2}$ Serviço de Cirurgia do Joelho, Hospital São Vicente de Paulo, Passo
} \\ Fundo, RS, Brasil

\begin{abstract}
Endereço para correspondência Larry Rodrigues De Campos Júnior, Residente, Serviço de Ortopedia e Traumatologia, Hospital São Vicente de Paulo, Rua Uruguai, 2050, Centro, Passo Fundo, RS,
\end{abstract} \\ 99010-112, Brasil (e-mail: dr.larryjunior@gmail.com).
}

Rev Bras Ortop 2021;56(6):741-746.

\section{Resumo}

Palavras-chave

- artroplastia do joelho

- osteoartrite do joelho

- patela
Objetivo Avaliar e comparar clínica e funcionalmente pacientes submetidos a artroplastia total primária do joelho (ATJ) com preservação ou substituição da patela. Métodos Em um estudo transversal, foram avaliados e comparados os resultados funcionais, usando escores de Western Ontario and McMaster Universities (WOMAC, na sigla em inglês) e Lequesne, de 158 pacientes submetidos a artroplastia total primária o joelho (162 joelhos), sendo que em 81 joelhos a patela foi submetida a artroplastia e em 81 a superfície articular da patela foi preservada.

Resultados Não foram identificadas diferenças significativas quanto ao escore de Lequesne $(p=0,585)$, escore global de $\operatorname{WOMAC}(p=0,169)$, nem quanto às subdivisões deste quanto a rigidez $(p=0,796)$ e a capacidade funcional $(p=0,190)$. Evidenciou-se diferença significativa apenas quanto à subdivisão que avalia a dor no escore de WOMAC, sendo menor no grupo submetido a artroplastia patelar $(p=0,036)$.

Conclusão No presente estudo, não houve diferença na avaliação funcional em pacientes submetidos a substituição ou não da patela durante a cirurgia de artroplastia primária do joelho. Entretanto, os indivíduos nos quais a patela foi preservada relataram mais dor.

Trabalho desenvolvido no Serviço de Ortopedia e Traumatologia do Hospital São Vicente de Paulo, Passo Fundo, RS, Brasil.

recebido

19 de Abril de 2020

aceito

19 de Setembro de 2020

Publicado on-line

Março 31, 2021
DOI https://doi.org/

$10.1055 / \mathrm{s}-0040-1721838$ ISSN 0102-3616. (c) 2021. Sociedade Brasileira de Ortopedia e Traumatologia. All rights reserved.

This is an open access article published by Thieme under the terms of the Creative Commons Attribution-NonDerivative-NonCommercial-License, permitting copying and reproduction so long as the original work is given appropriate credit. Contents may not be used for commercial purposes, or adapted, remixed, transformed or built upon. (https://creativecommons.org/ licenses/by-nc-nd/4.0/)

Thieme Revinter Publicações Ltda., Rua do Matoso 170, Rio de Janeiro, RJ, CEP 20270-135, Brazil 


Abstract
Keywords
- arthroplasty,
replacement, knee
- osteoarthritis, knee
- patella

Objective To evaluate and compare clinically and functionally patients undergoing primary total knee arthroplasty (TKA) with preservation or replacement of the patella. Methods In a cross-sectional study, the functional results were evaluated and compared, using the Western Ontario and McMaster Universities (WOMAC) and Lequesne scores, of 158 patients undergoing primary TKA (162 knees); in 81 knees the patella was submitted to arthroplasty and in 81 the joint surface of the patella was preserved.

Results No significant differences were identified in terms of the Lequesne score $(p=0.585)$, global WOMAC score $(p=0.169)$, nor in terms of its subdivisions regarding stiffness $(p=0.796)$ and functional capacity $(p=0.190)$. There was a significant difference only in terms of the subdivision that evaluates pain in the WOMAC score, being lower in the group undergoing patellar arthroplasty $(p=0.036)$.

Conclusion In the present study, there was no difference in functional assessment in patients who underwent or not patellar replacement during primary knee arthroplasty surgery. However, individuals in whom the patella was preserved reported more pain.

\section{Introdução}

A artroplastia total de joelho (ATJ) é uma cirurgia de eficácia clínica comprovada ${ }^{1-4}$ associada a substancial melhora funcional e na qualidade de vida em indivíduos portadores de gonartrose. .,6 $^{5}$

A primeira prótese total do joelho (PTJ) moderna foi desenvolvida pelo ortopedista canadense Frank Guston em 1960, sendo posteriormente aprimorada por John Insall nos anos 70, com o componente patelar apresentado por Townley e Insall em 1972. ${ }^{7}$ A introdução do componente patelar nas ATJ reduziu a ocorrência de dor anterior no joelho; no entanto, trouxe consigo novas complicações como a falha do componente, instabilidade, fratura patelar, ruptura tendínea do mecanismo extensor, necrose avascular da patela e outras lesões de tecidos moles. ${ }^{8}$ Ainda que essas complicações tenham sido atribuídas à má técnica cirúrgica e ao posicionamento inadequado do implante, o medo de sustentá-las tornou proibitiva a adoção da substituição da patela como rotina. ${ }^{8}$

Com o passar dos anos, houve um aprimoramento das técnicas cirúrgicas, as quais, associadas à evolução dos implantes, geraram significativa redução na ocorrência de complicações nas artroplastias patelares, dividindo os cirurgiões no que diz respeito à rotina por eles adotada frente à sua realização. ${ }^{9}$ Alguns autores sugerem realizar a substituição da patela rotineiramente devido à melhora da dor, melhores escores funcionais, maior satisfação do paciente e menor taxa de reintervenção por persistência da dor. ${ }^{10}$ Outros defendem a preservação da patela como rotina, por conta do risco das complicações, e apontam ainda como vantagem a manutenção do estoque ósseo da patela e taxas de satisfação e função similares. ${ }^{11-13}$ Adicionalmente, ainda há aqueles que defendem a substituição patelar em casos selecionados e acreditam que a realização de rotina não é sustentada pela literatura. ${ }^{14}$

0 presente estudo tem como objetivo avaliar funcionalmente indivíduos submetidos a ATJ primária do joelho com a substituição da superfície articular da patela e compará-los a indivíduos em quem a superfície articular da patela foi preservada.

\section{Materiais e Métodos}

Um total de 191 pacientes submetidos a ATJ primária de janeiro de 2012 a dezembro de 2014 por gonartrose primária foram inicialmente selecionados em nosso banco de dados para participarem do estudo. Todos tinham pelo menos 5 anos de seguimento, usaram o mesmo implante (Advance Medial-Pivot Knee System, Microport Orthopaedics, Arlington, TN, EUA) e foram operados por dois experientes cirurgiões de um hospital de referência em ensino ortopédico. Após a análise de prontuários, foram excluídos 33 pacientes, 7 por óbitos, 2 que estavam impossibilitados por comorbidades não relacionadas à ATJ, e 24 por abandono de seguimento. Nenhum paciente se encaixou no critério de exclusão referente à presença de complicações com grande prejuízo funcional. Por fim, 158 pacientes foram incluídos no estudo, 4 dos quais submetidos a ATJ bilateral em diferentes anos; dentre esses, 2 tiveram um joelho submetido a artroplastia patelar e 1 cuja patela foi preservada; 1 teve a patela de ambos joelhos preservadas e outro teve ambas submetidas a artroplastia. Nesses casos, ambos os joelhos foram avaliados separadamente. A amostra final constou de 162 joelhos. Em 81 joelhos, a patela foi substituída, e em 81 joelhos a superfície articular da patela foi preservada.

Os joelhos foram divididos em dois grupos: aqueles nos quais foi realizada a substituição da patela durante a ATJ e os nos quais a superfície articular da patela foi preservada. A substituição da patela foi indicada de maneira seletiva, sendo realizada na presença de artrose femoropatelar moderada e grave, mediante avaliação macroscópica no transoperatório; apenas patelas com superfície articular em boas condições foram preservadas.

Todos os pacientes foram contatados através de ligação telefônica realizada por um dos autores (de Campos Júnior L. 
R.) e responderam aos questionários de Lequesne ${ }^{15}$ e Western Ontario and McMaster Universities (WOMAC), ${ }^{16}$ este último com suas subdivisões. Durante o contato, os indivíduos foram convidados a participar do estudo e após a concordância a ligação era continuada.

Os pacientes que aceitaram participar do estudo assinaram o Termo de Consentimento Livre e Esclarecido (TCLE), sendo o estudo previamente aprovado pelo Comitê de Ética em Pesquisa da instituição.

\section{Análise Estatística}

As variáveis quantitativas foram descritas por média e desvio padrão (DP) ou mediana e amplitude interquartílica. As variáveis categóricas foram descritas por frequências absolutas e relativas. Para comparar médias, o teste t de Student foi aplicado, sendo que em caso de assimetria aplicou-se o teste de Mann-Whitney, e na comparação de proporções fezse valer do teste qui-quadrado de Pearson. O nível de significância adotado foi de $5 \%(\mathrm{p}<0,05)$, e as análises foram realizadas no programa IBM SPSS Statistics for Windows, versão 25.0 (IBM Corp. Armonk, NY, EUA).

\section{Resultados}

Dentre os pacientes, 46 pertenciam ao sexo masculino e 112 ao sexo feminino; os grupos de estudo são homogêneos tanto na distribuição dos gêneros quanto no que diz respeito ao número de joelhos (- Tabela 1). Também houve homogeneidade no que se refere à idade dos pacientes no ano em que a cirurgia foi realizada, na data da aplicação dos questionários, assim como no intervalo entre elas ( $\boldsymbol{- T}$ Tabela 2 ).

Em relação ao escore de Lequesne, o grupo que teve a patela preservada obteve mediana de 3,5 pontos, e o que teve

Tabela 1 Distribuição dos joelhos quanto a artroplastia ou preservação e gênero

\begin{tabular}{|l|l|l|l|}
\hline & Masculino & Feminino & Total \\
\hline Preservação da Patela & 23 & 58 & 81 \\
Artroplastia Patelar & 24 & 57 & 81 \\
\hline & 47 & 115 & 162 \\
\hline
\end{tabular}

Tabela 2 Caracterização da amostra quanto a idade e seguimento

\begin{tabular}{|l|l|l|l|}
\hline Variáveis & $\begin{array}{l}\text { Sem } \\
\text { substituição } \\
\text { da patela* }\end{array}$ & $\begin{array}{l}\text { Com } \\
\text { substituição } \\
\text { da patela* }\end{array}$ & valor-p** \\
\hline $\begin{array}{l}\text { Idade na } \\
\text { cirurgia } \\
\text { (anos) }\end{array}$ & $71,9 \pm 9,5$ & $71,3 \pm 6,3$ & 0,614 \\
\hline $\begin{array}{l}\text { Idade na } \\
\text { coleta (anos) }\end{array}$ & $77,9 \pm 9,8$ & $77,2 \pm 6,4$ & 0,595 \\
\hline $\begin{array}{l}\text { Seguimento } \\
\text { (anos) }\end{array}$ & $5,9 \pm 0,7$ & $5,9 \pm 0,8$ & 0,918 \\
\hline
\end{tabular}

*Média \pm Desvio Padrão.

${ }^{*}$ Diferença significativa estatisticamente considerando $p<0,05$.
Tabela 3 Avaliação dos escores conforme os grupos

\begin{tabular}{|l|l|l|l|}
\hline Escores & $\begin{array}{l}\text { Sem } \\
\text { substituição } \\
\text { da patela }\end{array}$ & $\begin{array}{l}\text { Vom } \\
\text { substituição } \\
\text { da patella }\end{array}$ & \multirow{2}{*}{ valor * $^{* *}$} \\
\cline { 2 - 3 } & $\begin{array}{l}\text { mediana } \\
\text { (P25-P75) }\end{array}$ & $\begin{array}{l}\text { mediana } \\
\text { (P25-P75) }\end{array}$ & \\
\hline $\begin{array}{l}\text { Lequesne } \\
\text { WOMAC }\end{array}$ & $3,5(0,5-7)$ & $2,5(0,5-7)$ & 0,585 \\
\hline Dor & $0(0-2)$ & $0(0-1)$ & $0,036^{* *}$ \\
\hline Rigidez & $0(0-0)$ & $0(0-0)$ & 0,796 \\
\hline $\begin{array}{l}\text { Atividade } \\
\text { Física }\end{array}$ & $7(1-12,5)$ & $5(0-9)$ & 0,190 \\
\hline Total & $8(2-15)$ & $5(0,5-11,5)$ & 0,169 \\
\hline
\end{tabular}

${ }^{* *}$ Diferença significativa estatisticamente considerando $p<0,05$.

a patela substituída obteve mediana de 2,5 pontos, diferença que não se mostrou suficiente para gerar significância (-Tabela 3).

Ao se analisar a escala de WOMAC, nas 3 áreas avaliadas (dor, rigidez e dificuldade para realização das atividades diárias/física) e escore global, observou-se diferença significativa apenas quanto ao quesito dor, cuja pontuação foi maior no grupo que teve a patela preservada ( - Tabela $3 \mathrm{e}-$ Figura $\mathbf{1}$ ). Não houve diferença significativa nos escores da avaliação da rigidez articular entre os grupos ( - Tabela 3 ). Embora não tenha se evidenciado diferença significativa quanto ao escore das dificuldades na realização das atividades de vida diária/capacidade física $(p=0,190)$ e o escore global $(p=0,169)$, observa-se pela mediana, P25 e P75 a presença de escores pouco maiores entre pacientes que tiveram a patela preservada ( - Tabela 3 e - Figuras 2 e $\mathbf{3}$ ). Devido ao fato dos escores serem expressos por números naturais, alguns deles apresentaram mediana igual a zero, o que decorre da maioria dos indivíduos ter obtido tal pontuação na área analisadas.

Três pacientes apresentaram complicações no decorrer do estudo: dois tiveram deiscência da ferida operatória, sendo que um havia sido submetido a artroplastia patelar e outro teve a patela preservada. Ambos foram submetidos a desbridamento cirúrgico e tratamento com antibióticos com boa evolução; o outro teve infecção periprotética precoce, sendo submetido a novo procedimento cirúrgico para troca do polietileno, lavagem e desbridamento, e tratamento com antibioticoterapia. Uma paciente excluída por abandono do seguimento teve infecção tardia no joelho, sendo submetida à amputação do membro.

\section{Discussão}

Ao longo dos anos, foram realizados diversos estudos visando avaliar a diferença de desfecho entre a realização ou não da artroplastia patelar. Dentre os mais recentes, Ha et al. ${ }^{17}$ realizaram o primeiro estudo prospectivo randomizado em que os pacientes tiveram um dos joelhos submetidos a ATJ com artroplastia patelar e outro com preservação no mesmo momento cirúrgico e usando o mesmo modelo de implante. Após 5 anos de seguimento, 60 pacientes ( 120 joelhos) foram 


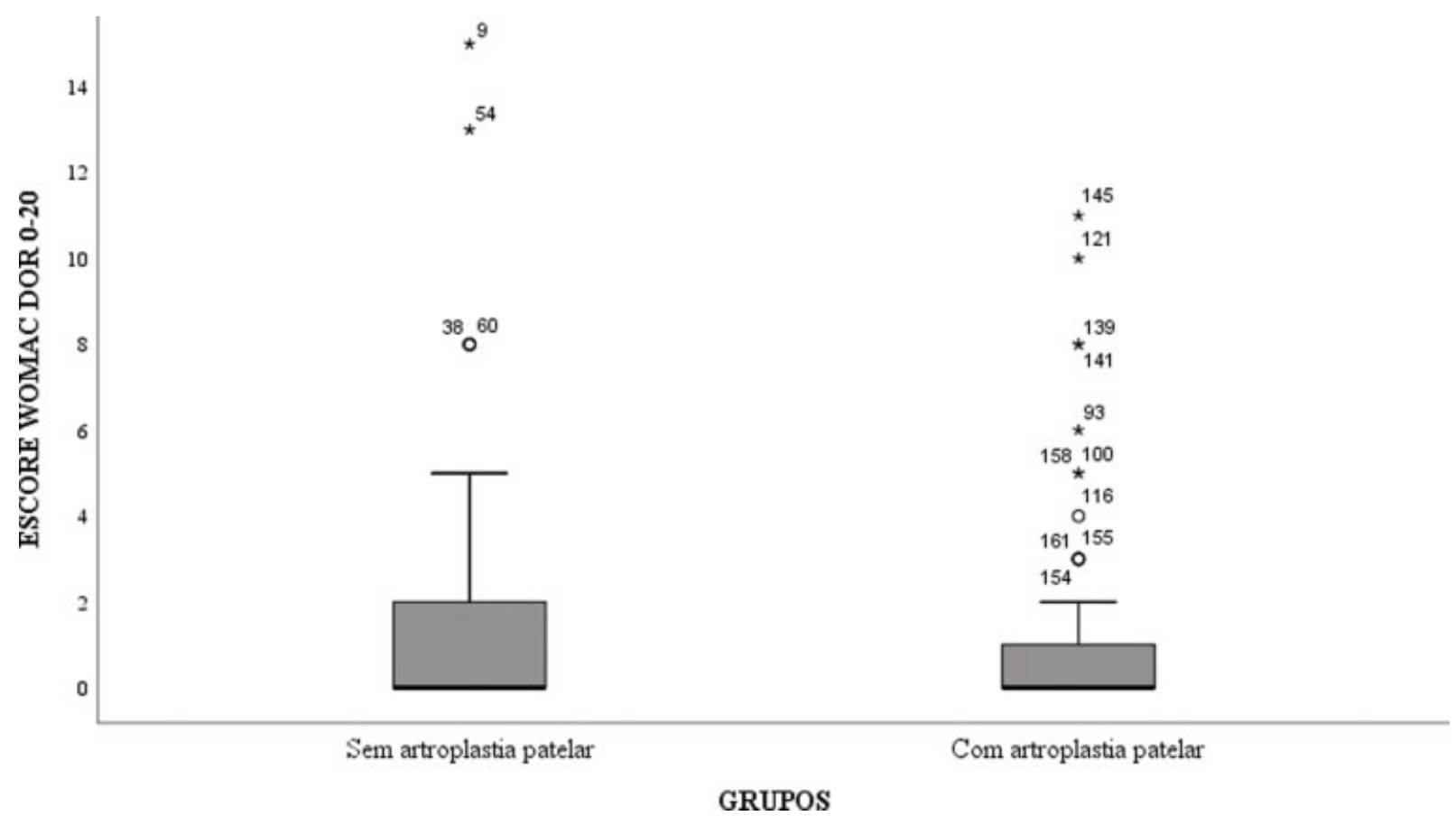

Fig. 1 Escore de WOMAC quanto à dor.

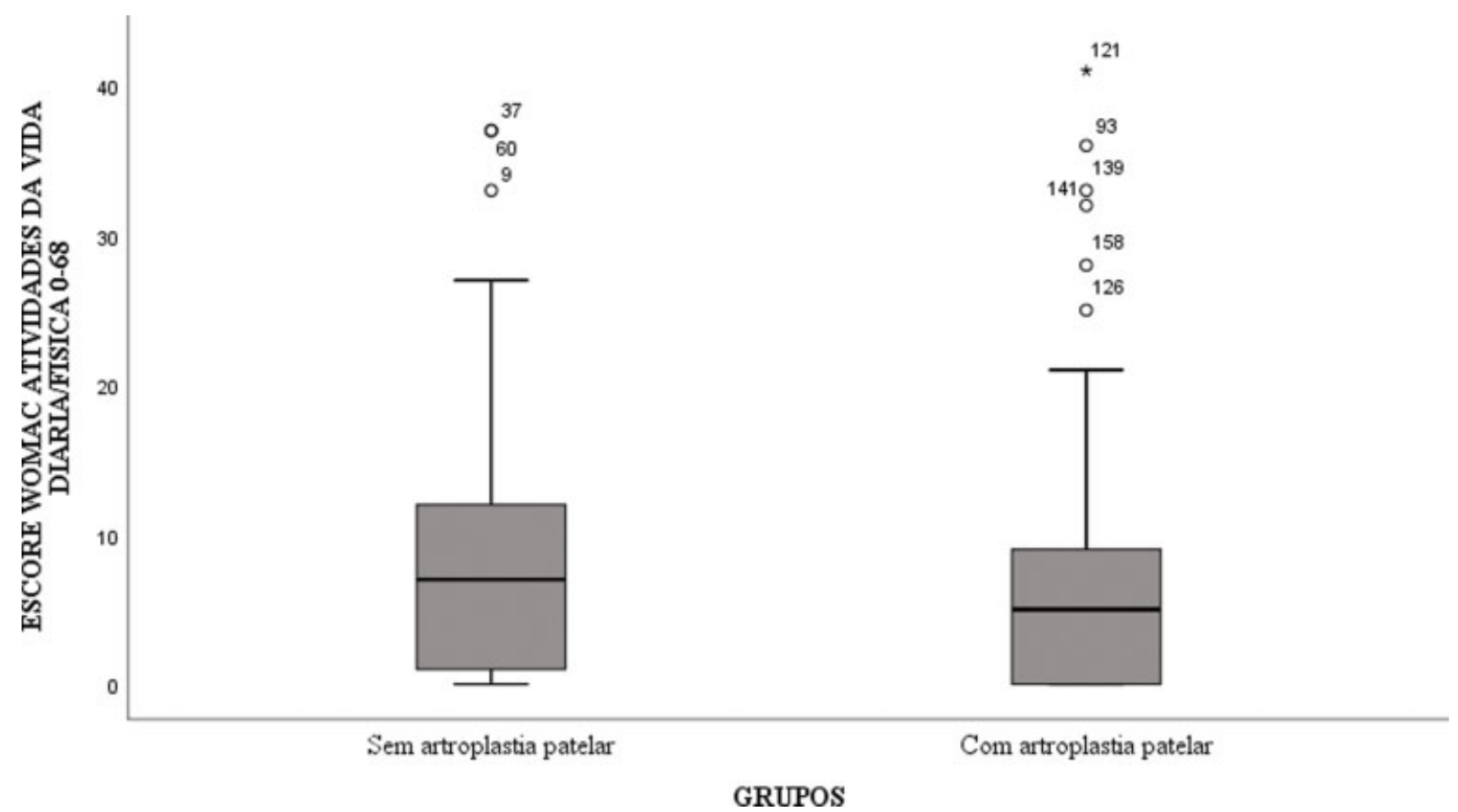

Fig. 2 Escore de WOMAC quanto à dificuldade nas atividades da vida diária.

reavaliados, e os autores constataram que os joelhos submetidos a artroplastia patelar apresentaram menor dor na face anterior $(p<0,001)$ e menor incidência de crepitação patelar $(\mathrm{p}<0,001)$. Ambos os joelhos apresentaram melhora funcional; no entanto, a mesma foi significativamente superior nos joelhos submetido a artroplastia patelar $(\mathrm{p}<0,001)$, e nenhum joelho apresentou complicações nem necessidade de revisão. A avaliação quanto à preferência dos pacientes mostrou que $47 \%$ preferiram o joelho da artroplastia patelar e $46 \%$ foram indiferentes. ${ }^{17}$
Migliorini et al. ${ }^{18}$ realizaram uma metanálise de 31 artigos, totalizando 4.132 joelhos, e Longo et al $^{19}$ realizou outra metaanalise de 35 artigos e um total de 5,535 joelhos. Ambos constataram menor dor na face anterior do joelho entre pacientes submetidos a artroplastia patelar $(\mathrm{p}=0,02 \mathrm{e}$ $\mathrm{p}=0,00001$ ) e menores taxas de revisão no mesmo grupo ( $\mathrm{p}<0,0001$ e $(\mathrm{p}=0,00001$, respectivamente). Apenas os primeiros evidenciaram diferença funcional estatisticamente significativa, com função melhor em pacientes submetidos a artroplastia patelar $(\mathrm{p}=0,009)$. 


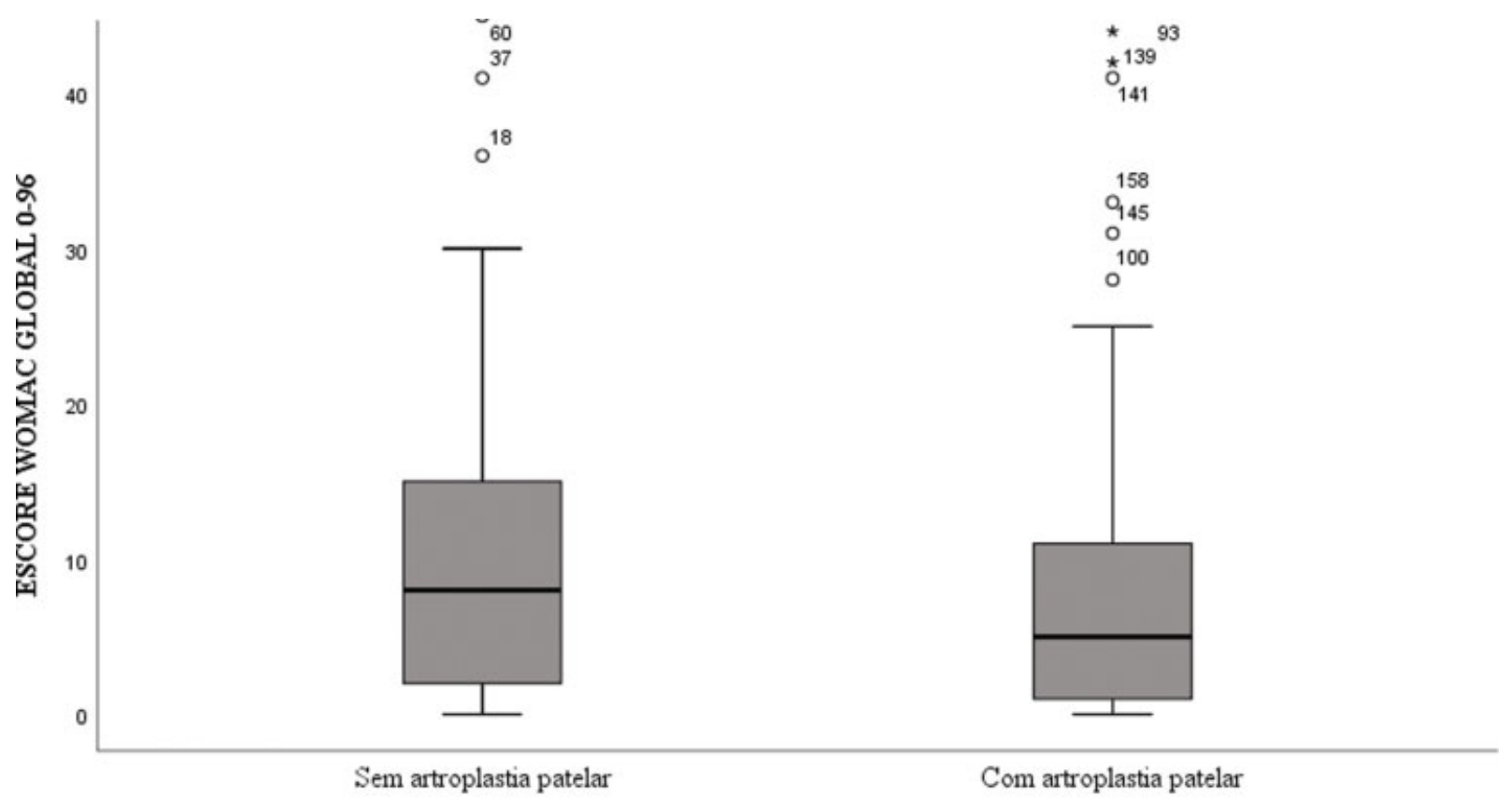

GRUPOS

Fig. 3 Escore WOMAC global.

Melhores desfechos quanto à dor em pacientes submetidos a artroplastia patelar foram o achado comum entre o nosso e os demais estudos apresentados, e nos parece um desfecho bem definido. Apesar de não termos evidenciado diferença significativa quanto ao WOMAC referente às atividades da vida diária/física e global, a presença de medianas maiores no grupo de preservação da patela e os percentis $25 \mathrm{e}$ 75 relativamente mais elevados, associados ao valor-p consideravelmente baixo, nos leva a crer que a diferença poderia tornar-se significativa caso a população do estudo fosse maior. Tal hipótese nos parece plausível, haja vista a significância evidenciada por Migliorini et al. ${ }^{18}$ e Ha et al., ${ }^{17}$ bem como o achado semelhante ao nosso, evidenciado por Longo et al., ${ }^{19}$ que acreditam haver tendência à melhor função quando se realiza a artroplastia patelar. Certamente, são necessários mais estudos voltados para a comparação funcional entre esses grupos de pacientes para que tenhamos respostas concretas quanto a isso.

Os três estudos evidenciaram menores taxas de revisão no grupo da artroplastia patelar. ${ }^{17-19}$ Migliorini e et al. ${ }^{18}$ acreditam que isso esteja relacionado à insatisfação dos indivíduos pela permanência da dor quando a patela é preservada, levando os cirurgiões a reoperarem para realizar a artroplastia patelar. ${ }^{18} \mathrm{Em}$ nosso trabalho, no entanto, buscamos avaliar apenas os resultados funcionais nos dois cenários em situações ideais, motivo pelo qual um dos fatores de exclusão definido foi a presença de complicações que levaram a grande prejuízo funcional. Acreditamos que a presença e análise dos mesmos afetaria de maneira não representativa os resultados no grupo ao qual pertencessem. Ressaltamos que apesar do definido, apenas uma paciente que foi excluída por abandono do seguimento apresentou complicação grave e de causa não atribuível à artroplastia patelar. Acreditamos que o desfecho relacionado à incidência de revisão não deve ser considerado, uma vez que há maior tendência de indicação de revisão nos pacientes sintomáticos em quem a patela foi preservada. Podemos observar alguns pontos questionáveis nas metanálises anteriormente citadas: falta da descrição da habilidade dos cirurgiões, do modelo de implante na maioria dos estudos e heterogeneidade dentre os estudos, ${ }^{19}$ uso de diferentes implantes e inclusão de estudos com 2 anos de seguimento. ${ }^{18}$

Zmistowski et al., ${ }^{20} \mathrm{em}$ um estudo de custo-efetividade, realizaram uma revisão de 14 estudos prospectivos randomizados que investigaram diferentes desfechos entre cirurgiões que optaram pela artroplastia patelar de forma seletiva e não seletiva. Entre os que realizaram a artroplastia patelar de forma não seletiva, a persistência de dor anterior do joelho foi de $20,9 \%$ no grupo de preservação e de $13,2 \%$ no grupo da artroplastia patelar $(\mathrm{p}<0,001)$, e as taxas de reoperação por patologias relacionadas à patela de 3,7 e $1,6 \%(\mathrm{p}<0,01)$, respectivamente. Nos estudos que excluíram as patelas com artrose, a incidência de dor anterior foi equivalente nos grupos, $3,1 \%$ no grupo da preservação e $3,2 \%$ no grupo artroplastia $(p=0,97)$; a taxa de reoperação por persistência da dor por conta da patela caiu para 1.2 e $0 \%(p=0,06)$, respectivamente. Avaliados os desfechos, complicações e custos relacionados, o estudo concluiu que a substituição da patela de forma rotineira não é o melhor custo benefício, mas sim a realização seletiva, por evitar revisões por persistência da dor, além de minimizar riscos inerentes à artroplastia patelar. ${ }^{20}$

Considerando que, em nosso serviço, realizamos a artroplastia patelar de forma seletiva, acreditamos que, hipoteticamente, expusemos o grupo de preservação patelar à melhor perspectiva possível em termos de resultados clínicos; afinal, por preservarmos apenas patelas em boas 
condições macroscópicas, podemos afirmar que os escores maiores do WOMAC quanto à dor no grupo que teve a patela preservada não decorreram de patelas com artrose moderada ou avançada. No entanto, mesmo em vigência da artroplastia seletiva, a diferença entre os grupos foi significativa, diferente do encontrado por Zmistowski et al. ${ }^{20}$ Acreditamos que a seleção também pode ter sido motivo de não identificarmos diferença significativa entre os grupos quanto ao escore WOMAC global e das atividades da vida diária, devido à provável redução na real diferença que poderia haver caso a seleção não ocorresse.

Reconhecemos limitações em nosso estudo, como o tamanho da amostra, falta de avaliação de escores de qualidade de vida e saúde mental, e a aplicação dos questionários por ligação telefônica. Merecem destaque o fato de que todos os pacientes tiveram o mesmo modelo de prótese implantada e o procedimento tenha sido realizado pelo mesmo grupo de cirurgiões experientes. Por termos realizado avaliação por escores validados para a população brasileira, ${ }^{15,16}$ a avaliação clínica e funcional baseou-se apenas em critérios subjetivos relatados pelos pacientes, impedindo a valorização de dados objetivos pelo avaliador. Acreditamos que isso traz resultados confiáveis, uma vez que, conforme Epstein, ${ }^{21}$ a sintomatologia referida pelo paciente sempre é o mais importante. Instrumentos baseados no que é relatado pelo paciente podem fornecer dados que não são conseguidos por avalições fisiológicas e que podem ter maior reprodutividade sobre a qualidade de vida do que índices clínicos, bioquímicos e fisiológicos. ${ }^{21}$

\section{Conclusão}

No presente estudo, não se evidenciou diferença significativa entre o grupo submetido a artroplastia patelar e o que teve a patela preservada quanto aos escores de Lequesne e subáreas do WOMAC das dificuldades para atividades da vida diária/atividade física, rigidez e global. Houve diferença significativa apenas quanto ao quesito dor do WOMAC, com desfecho melhor no grupo submetido a artroplastia patelar mesmo na vigência da artroplastia seletiva.

\section{Suporte Financeir}

Não houve suporte financeiro de fontes públicas, comerciais, ou sem fins lucrativos.

\section{Conflito de Interesses}

Os autores declaram não haver conflito de interesses.

\section{Referências}

1 Harwin SF, Greene KA, Hitt K. Triathlon total knee arthroplasty: 4year outcomes with a high-performance implant. J Knee Surg 2008;21(04):320-326

2 Bourne RB. Measuring tools for functional outcomes in total knee arthroplasty. Clin Orthop Relat Res 2008;466(11):2634-2638
3 Parsley BS, Conditt MA, Bertolusso R, Noble PC. Posterior cruciate ligament substitution is not essential for excellent postoperative outcomes in total knee arthroplasty. J Arthroplasty 2006;21(06, Suppl 2):127-131

4 Kane RL, Saleh KJ, Wilt TJ, Bershadsky B. The functional outcomes of total knee arthroplasty. J Bone Joint Surg Am 2005;87(08): $1719-1724$

5 Ogrodzka K, Chwała W, Niedźwiedzki T. Three-dimensional pattern of knee movement in patients with gonarthrosis. Ortop Traumatol Rehabil 2007;9(06):618-626

6 Leão MGS, Santoro ES, Avelino RL, Coutinho LI, Granjeiro RC, Orlando N Junior. Avaliação da qualidade de vidas em pacientes submetidos a artroplastia total de joelho. Rev Bras Ortop 2014;49 (02):194-201

7 Papas PV, Cushner FD, Scuderi GR. The history of total knee arthroplasty. Tech Orthop 2018;33(01):2-6

8 Hsu RW. The management of the patella in total knee arthroplasty. Chang Gung Med J 2006;29(05):448-457

9 Swan JD, Stoney JD, Lim K, Dowsey MM, Choong PF. The need for patellar resurfacing in total knee arthroplasty: a literature review. ANZ J Surg 2010;80(04):223-233

10 Maffulli N, Longo UG, Gougoulias N, Caine D, Denaro V. Sport injuries: a review of outcomes. Br Med Bull 2011;97(01):47-80

11 Garneti N, Mahadeva D, Khalil A, McLaren CA. Patellar resurfacing versus no resurfacing in Scorpio total knee arthroplasty. J Knee Surg 2008;21(02):97-100

12 Pakos EE, Ntzani EE, Trikalinos TA. Patellar resurfacing in total knee arthroplasty. A meta-analysis. J Bone Joint Surg Am 2005;87 (07):1438-1445

13 Meding JB, Fish MD, Berend ME, Ritter MA, Keating EM. Predicting patellar failure after total knee arthroplasty. Clin Orthop Relat Res 2008;466(11):2769-2774

14 Allen W, Eichinger J, Friedman R. Resurfaced versus non-resurfaced patella in total knee arthroplasty. J Knee Surg 2019;32(07): 611-615

15 Marx FC, Oliveira LM, Bellini CG, Ribeiro MCC. Tradução e validação cultural do questionário algo funcional de Lequesne para osteoartrite de joelhos e quadris para a língua portuguesa. Rev Bras Reumatol 2006;46(04):253-260

16 Fernandes MI. Tradução e validação do questionário de qualidade de vida específico para osteoartrose WOMAC (Western Ontario McMaster Universities) para a língua portuguesa [tese]. São Paulo: Universidade Federal de São Paulo, Escola Paulista de Medicina; 2003

17 Ha C, Wang B, Li W, Sun K, Wang D, Li Q. Resurfacing versus notresurfacing the patella in one-stage bilateral total knee arthroplasty: a prospective randomized clinical trial. Int Orthop 2019; 43(11):2519-2527

18 Migliorini F, Eschweiler J, Niewiera M, El Mansy Y, Tingart M, Rath B. Better outcomes with patellar resurfacing during primary total knee arthroplasty: a meta-analysis study. Arch Orthop Trauma Surg 2019;139(10):1445-1454

19 Longo UG, Ciuffreda M, Mannering N, D’Andrea V, Cimmino M, Denaro V. Patellar resurfacing in total knee arthroplasty: systematic review and meta-analysis. J Arthroplasty 2018;33(02): 620-632

20 Zmistowski BM, Fillingham YA, Salmons HI, Ward DT, Good RP Lonner JH. Routine patellar resurfacing during total knee arthroplasty is not cost-effective in patients without patellar arthritis. J Arthroplasty 2019;34(09):1963-1968

21 Epstein AM. The outcomes movement-will it get us where we want to go? N Engl J Med 1990;323(04):266-270 